\title{
A p-DIMETHYLAMINOBENZALDEHYDE-NITRITE METHOD FOR THE HISTOCHEMICAL DEMONSTRATION OF TRYPTOPHANE AND RELATED COMPOUNDS
}

\author{
BY \\ C. W. M. ADAMS \\ From the Bernhard Baron Institute of Pathology, The London Hospital
}

(RECEIVED FOR PUBLICATION APRIL 14, 1956)

Ehrlich's reagent (p-dimethylaminobenzaldehyde) has been extensively used for the biochemical detection of indole derivatives; correspondingly a histochemical method, incorporating this reagent, has been described by Lison (1936) for the demonstration of these compounds in tissues. In this method a reddish-violet pigment is formed by the condensation of p-dimethylaminobenzaldehyde (henceforth abbreviated to DAB) with the indole nucleus. However, this method lacks selectivity, since many other organic reagents are able to react with DAB to form red or yellow condensation products. This technique also lacks sensitivity, since, in spite of the wide distribution of the indole derivative tryptophane in the tissues, moderate to strong reactions are only given with fair consistency by Russell bodies and only irregularly by the layers of Huxley and Henle of the hair-shaft sheath (Pearse, 1953). Several other aldehyde techniques for the histochemical demonstration of tryptophane have been introduced, such as the Romieu reaction (Blanchetière and Romieu, 1931), a modification of the VoisenetFürth reaction (Serra, 1946), and an adaptation of the Bates reaction introduced by Kaufmann, McDonald, and Gay (1946) and Kaufmann, McDonald, Gay, Wilson, and Wyman (1947). In this last method DAB was employed with the addition of sodium nitrate as an oxidizing agent. However, none of these techniques was recommended by Pearse (1953), since the colour formed was unstable or, as well, too faint for accurate study.

In a different field DAB has been extensively employed for the biochemical estimation of either free or protein-bound tryptophane. In this colorimetric method a bright blue pigment is formed when condensation occurs between DAB and tryptophane, or certain other indole derivatives, in the presence of an oxidizing agent such as hydrogen peroxide, sodium nitrite, sodium nitrate, or copper sulphate (Portner and Högl, 1953). These authors recommended a method which employed nitrite as an oxidizing agent (Spies and Chambers, 1948). This blue pigment was reported to be formed only from indole derivatives, such as tryptophane, with an attached side-chain at the " 3 " position, leaving the " 1 " and " 2 " positions of the pyrrole ring free (Giral and Laguna, 1950 ; Portner and Högl, loc. cit.). It has been suggested that the blue pigment may, in the case of indoles of similar structure to tryptophane, be an oxidation product of a $\beta$-carboline (norharman) derivative formed by condensation and ring-closure of the indole with aldehydes (Fig. 1). This has been partly confirmed by Harvey, Miller, and Robson (1941), who showed that the characteristic blue pigment ("carboline-blue") could be formed by the oxidation of $2: 3: 4: 5$-tetrahydro- $\beta$-carboline4-carboxylic acid, a colourless crystalline compound prepared by the interaction of formaldehyde and tryptophane. However, such a mechanism does not explain the formation of blue pigments from those 3-indolyl derivatives such as 3-methylindole and 3-indolylacetic acid, which possess insufficient carbon atoms in their side chain to complete the third ring (Fig. 1) of the $\beta$-carboline of nucleus. Nevertheless, in spite of the inexact $\tilde{O}$ knowledge of the mechanism of this reaction, the $\mathbb{E}$ appearance of a blue pigment is regarded as a 0 specific test for the detection of 3-indolyl derivatives.

On account of the specificity of this method for $\stackrel{\odot}{+}$ the demonstration of these compounds this paper $\underline{T}$ is concerned with the histochemical adaptation of such a method, employing DAB and an oxidizing agent, in particular sodium nitrite as recommended $\mathbb{\Phi}$ by Portner and Högl (loc. cit.). Although all 3indolyl derivatives would be expected to react with this DAB-nitrite method, only tryptophane and 


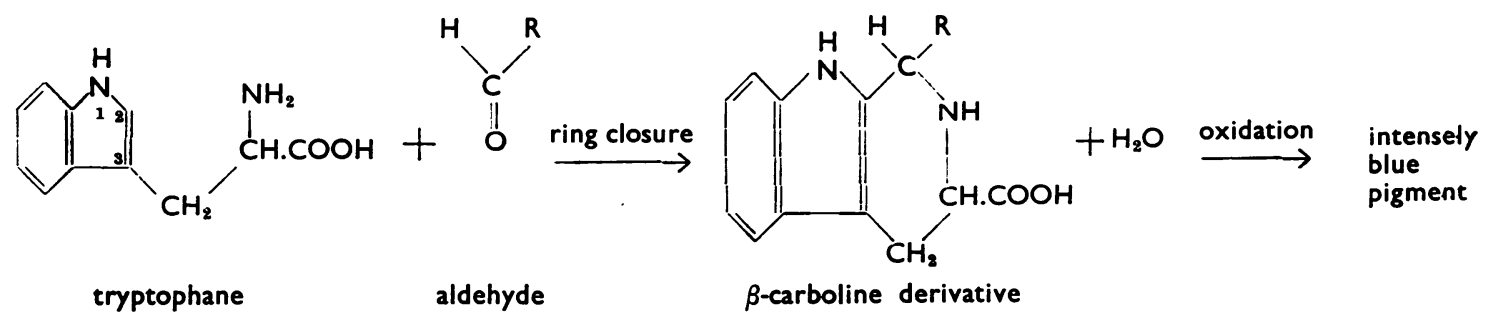

Fig. 1. - Suggested reaction between tryptophane and aldehydes in the presence of an oxidizing agent (modified from Portner and Högl).

possibly serotonin need be considered, since other soluble tissue-indoles, which are not bound to protein, would not survive ordinary embedding processes.

\section{Methods}

In Vitro. - The reactions were examined between DAB and certain indole derivatives, amino-acids, and a variety of compounds known to react with Ehrlich's reagent. The following substances were tested: dltryptophane, tryptamine, serotonin creatinine sulphate, 3-indolylacetic acid, indole, cysteine, histidine, tyrosine, proline, lysine, arginine, serine, glycine, phenol, orcin, phlorglucinol, resorcinol, pyrocatechol, hydroquinone, $\alpha$-naphthol, methylamine, aniline, benzidine, $\beta$-naphthylamine, phenylhydrazine, aminobenzoic acid, sulphanilic acid, pyrrole, pyridine, uric acid, and creatinine.

All the reagents were prepared at a strength of about $1 \mathrm{~g}$. per $100 \mathrm{ml}$.; when insoluble in water they were dissolved in $70 \%$ ethyl alcohol. A solution of DAB ( $5 \mathrm{~g}$. per $100 \mathrm{ml}$.) in concentrated hydrochloric acid (S.G. 1.18) was prepared and $9 \mathrm{ml}$. was added to $1 \mathrm{ml}$. quantities of the solution of each substance tested. In each case the colour of any reaction product with DAB was noted, and any change of colour induced by oxidation with $0.05 \%$ sodium nitrite or nitrate was observed.

Histochemical Applications.-The tissues examined, obtained from the rat, human post-mortem and human surgical material, were usually fixed in $1 \%$ trichloracetic acid in $80 \%$ ethyl alcohol ; occasionally fixation in $10 \%$ sulphosalicylic acid or $70 \%$ methylated alcohol was employed. After embedding in paraffin wax, sections were cut at $15 \mu$, floated on to " albuminized," chromic acid-cleaned slides and then subjected to the following procedure:

(1) The sections were brought to absolute alcohol and then allowed to become just dry in the air at room temperature. As an alternative, sections were coated, after removal from alcohol, with a thin film of celloidin $(0.25 \%)$, which was not allowed to become more than just dry before proceeding to the next stage.

(2) Next the sections were immersed in a $5 \%$ solution of DAB in concentrated hydrochloric acid (S.G. 1.18) for one minute.

(3) The sections were then transferred to a $1 \%$ solution of sodium nitrite in concentrated hydrochloric acid for a further period of one minute.
(4) Finally the sections were rinsed in absolute alcohol, washed in running tap-water for about half a minute, dehydrated in alcohol, and mounted in conventional mounting media.

In order to verify that this technique embodied the best combination of reagents, applied in the most suitable order, for the development of maximum colour in tissues, the following alterations were made to the basic method:

(a) The use of sodium nitrate as an oxidizing agent in stage $3 ;(b)$ the use of 40 and $70 \%$ hydrochloric acid as a solvent for the DAB reagent in stage 2 ; (c) the addition of nitrite to the DAB reagent in stage 2 , with the omission of stage 3 ; (d) preliminary subjection of tissue sections to oxidation by nitrite between stages 1 and 2, with the omission of stage 3 . Since it was expected that the DAB-nitrite reaction would be weaker after formalin fixation, some tissues were fixed in $4 \%$ formaldehyde-saline for 12 hours and 14 days for comparison with alcohol-fixed tissues. The effect of chromate-containing fixatives was also examtned. With a view to preventing this DAB-nitrite reaction by preliminary blockade, some sections were subjected to oxidation by performic acid (prepared according to Pearse, 1953) before immersion in the reagent mixtures; it was expected that tryptophane would be unavailable for reaction with DAB after immersion in performic acid, since this acid has, among the commonly-occurring amino-acids, a selective oxidant effect on tryptophane, cystine. and methionine (Toennies and Homiller, 1942).

\section{Results}

In Vitro.- It was found that only 3-indolyl derivatives, amongst those compounds examined here, formed strong blue pigments after about $10 \mathrm{sec}$. oxidation of the DAB-condensation product by sodium nitrite. A change of colour, e.g. from red to yellow or brown to pink, was often noticed with these other compounds, but in no case was a blue colour formed. Indole itself was found to form a weak grey-violet pigment after more prolonged oxidation (15 min.).

Oxidation by sodium nitrite was more rapid and effective than by the use of nitrate, which required some $5 \mathrm{~min}$. action to produce the colour 
change brought about by $10 \mathrm{sec}$. exposure to nitrite. Atmospheric oxidation for a few hours was also able to form these blue pigments: thus solutions of 3-indolyl derivatives, absorbed on filter paper after reaction with DAB, slowly turned blue as they dried.

Histochemical Application.-The standard technique (vide supra) was found to give the most satisfactory and consistent results in tissues. Thus the use of sodium nitrate as an oxidizing agent was ineffective ; weaker strengths of hydrochloric acid led to a less intense reaction; the inclusion of nitrite in the DAB solution led to variable and inconsistent results. Further, if the strength of nitrite in this last modification was too high, or if the tissue was exposed to nitrite before DAB, then no colour was formed at all. Formalin and also chromate fixation was found to interfere with the subsequent demonstration of 3-indolyl derivatives in tissues by this DAB-nitrite method. This interference, in the case of formalin, was found to be only slight after 12 hours' fixation, but very marked after 14 days' fixation in $4 \%$ formaldehyde-saline. Preliminary oxidation of tissue sections in $90 \%$ performic acid, used as a blockading agent, prevented any subsequent reaction with DAB.

The following results were obtained in tissues (human, except where stated) : a strong reaction was found in fibrin, fibrinoid, Paneth cell granules, gastric peptic cell granules, pancreatic zymogen granules in the rat and man, thyroid colloid, all types of muscle fibres, neurokeratin of the myelin sheath of peripheral nerves in the rat, mouse, and man, and the inner hair-root sheath. A moderate reaction was given by the parenchymal cells of the liver, the stratum reticularis of the suprarenal, variably by both acidophil and basophil cells of the adenohypophysis, the cytop!asm of cells lining the periphery of lobules of a malignant argentaffinoma, hair keratin (only after alkaline hydrolysis), deeper layers of the epidermis, neurokeratin of the myelin of the central nervous system, astrocyte fibres, cytoplasm of the Purkinje cell, cytoplasm of neurones of the supraoptic, paraventricular, and mamillary nuclei, and cytoplasm of neurones in the posterior root ganglion. No reaction was obtained in collagen, reticulin, oxyntic cells, intestinal goblet cells, pancreatic islet-cells in the rat and man, melanin of the epidermis and of the substantia nigra, "lipofuscins" of the liver, suprarenal cortex, and of the central nervous system, follicular cells of the thyroid, posterior lobe of the pituitary in the rat and man, cytoplasm of the Betz cell and axis cylinders in the central nervous system and in peripheral nerves. Variable, often negative, results were obtained in elastic tissue and nucleoprotein.

\section{Discussion}

Three aspects of the DAB nitrite reaction have to be considered: (a) chemical specificity : (b) adaptation as a histochemical method; and (c) histochemical findings in tissues.

(a) Chemical Specificity.-From studies in vitro it was apparent that a blue reaction-product with $\mathrm{DAB}$, in the presence of nitrite, was formed by 3indolyl derivatives, such as tryptophane (3-indolylaminopropionic acid), tryptamine (3-indolylethylamine), serotonin (5-hydroxy-3-indolylethylamine), and 3-indolylacetic acid. Other amino-acids and organic compounds studied here formed reddish or yellow, but never blue, condensation products with DAB. These findings confirm those of Giral and Laguna (1950) and Portner and Högl (1953) that 3-indolyl derivatives are capable of forming blue oxidation products after condensation with DAB. It was found here, in accord with Spies and Chambers (1948) and Portner and Högl (1953), that the reaction of the 3-indolyl derivatives with DAB appeared to proceed in two stages, namely, (1) 3-indolyl derivative $+\mathrm{DAB} \rightarrow$ red condensation product, and (2) red condensation product + nitrite $\rightarrow$ intensely blue pigment. However, since the red condensation product of stage 1 became blue when allowed to become dry on paper, it may be inferred that atmospheric oxygen is able, although slowly, to effect the formation of the blue pigment of stage 2 .

(b) Histochemical Method.-It was found, after various manœuvres had been tried, that the best result was obtained with the standard technique, that is, tissue sections were first allowed to react with DAB (dissolved in concentrated hydrochloric acid) and then subsequently exposed to nitrite, a reagent which was found to be far more effective in forming the blue oxidation pigment than sodium nitrate. This view would appear to be contradictory to that of Kaufmann and his colleagues (1946, 1947), who were able to distinguish tryptophane-rich proteins from tryptophane-free histones by a similar technique, using weaker acid as a solvent for DAB and employing nitrate as an oxidizing agent. However, the method of these authors was found, here and by Pearse (1953), to give only feeble reactions ; moreover, it could well be argued that any success if impure samples of nitrate were employed might be due to contaminating traces of nitrite. 
Fixation in $1 \%$ trichloracetic acid in $80 \%$ ethyl alcohol was found to give the best results. Although fixation in $4 \%$ formaldehyde-saline for some 12 hours did not greatly reduce the depth of colour formed in the reaction, more prolonged exposure to this fixative led to a marked loss of intensity of the reaction. Thus it is likely that formalin, as an aldehyde, reacts with indoles in a similar way to DAB, but, in the absence of strong acids, more slowly and possibly incompletely. Likewise chromate-containing fixatives, probably on account of their strong oxidizing properties, almost completely prevented subsequent reaction with DAB. Therefore, in order to obtain consistent and strong reactions with this DAB-nitrite method alcoholic fixatives should be used rather than those containing formaldehyde. Chromatecontaining fixatives should never be employed.

Complete blockade of this DAB-nitrite reaction occurred after preliminary oxidation by performic acid. This is in accord with Danielli's $(1947,1950)$ use of this acid to prevent tryptophane in tissues reacting with tetrazotized benzidine (coupled alkaline tetrazonium method). The mechanism of this blockade may depend upon the oxidation of tryptophane by performic acid (Toennies and Homiller, 1942) to a compound, which these authors did not identify, but was found here, in vitro, to be a brownish-black pigment which, like melanin, failed to react with the DAB-nitrite method.

On relating the reactions obtained with DAB in tissues to those seen in vitro, on one hand it may be inferred that in the absence of nitrite the reaction only proceeds to stage 1 (vide supra). This stage probably represents that obtained in the indole reaction, as described by Lison (1936), in which no oxidizing agents were employed. On the other hand, in the presence of nitrite the reaction proceeds rapidly to stage 2 , with the production of a blue pigment of much greater intensity than the red or reddish-violet pigment formed in stage 1. However, if Lison's DAB-hydrochloric acid-ethyl alcohol mixture was applied, boiling, to alcohol-fixed tissue sections then irregular areas of faint blue as well as the usual reddish-violet pigments were seen. From this it is inferred that atmospheric oxidation at higher temperatures is able in tissues to effect, although incompletely, the formation of the blue pigment of stage 2 .

(c) Histochemical Findings.-In the tissues it was found for the most part that a strong reaction with DAB occurred at sites where, by biochemical analysis (see Table), a high concentration of tryptophane might be expected. However, there were
TABLE

TRYPTOPHANE CONTENT OF SOME TISSUES AND PROTEINS OBTAINED FROM VARIOUS ANIMAL SOURCES*

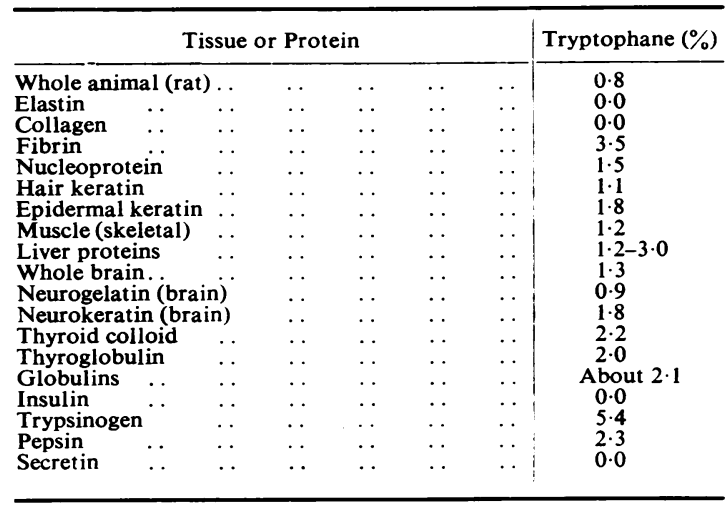

* From Block and Bolling, 1945.

some exceptions to this, namely, that elastic fibres were found to give a variable, rather weak reaction ; this result is surprising, since elastin, by biochemical analysis, contains no tryptophane. A further discrepancy was the presence of only a faint reaction in nucleoprotein, which contains $1.5 \%$ tryptophane. In this case the feeble reaction may in part be due to the solvent action of strong acids on chromatin, similar to that seen with unbuffered performic acid (Danielli, 1947). At first the keratin of the hair cortex was found to give no reaction for tryptophane after the application of the standard technique. However, this keratin after alkaline hydrolysis gave a moderately strong reaction. This suggests that, in the case of the keratin of hair, tryptophane was accessible to the DAB reagent only after preliminary rupture of its molecular structure, probably by destruction of disulphide bonds by alkaline hydrolysis (Cuthbertson and Phillips, 1945).

Although it cannot be argued that the presence of a strong histochemical reaction for 3-indolyl derivatives in, or around, a cell known to secrete a tryptophane-rich substance of necessity localizes this secretion at that point, it is of interest nevertheless that a strong reaction for tryptophane was found in pancreatic zymogen granules (Fig. 2) and the granules of gastric peptic cells. Both of these types of granules may be concerned in the formation of the tryptophane-rich (see Table) alimentary proteolytic enzymes (Bourne, 1951). Likewise a strong reaction with the DAB-nitrite method was found in the granules of the intestinal Paneth cells (Fig. 3). These cells also are concerned with the secretion of certain intestinal peptidases (Bourne, loc. cit.). The strong reaction found in the thyroid 


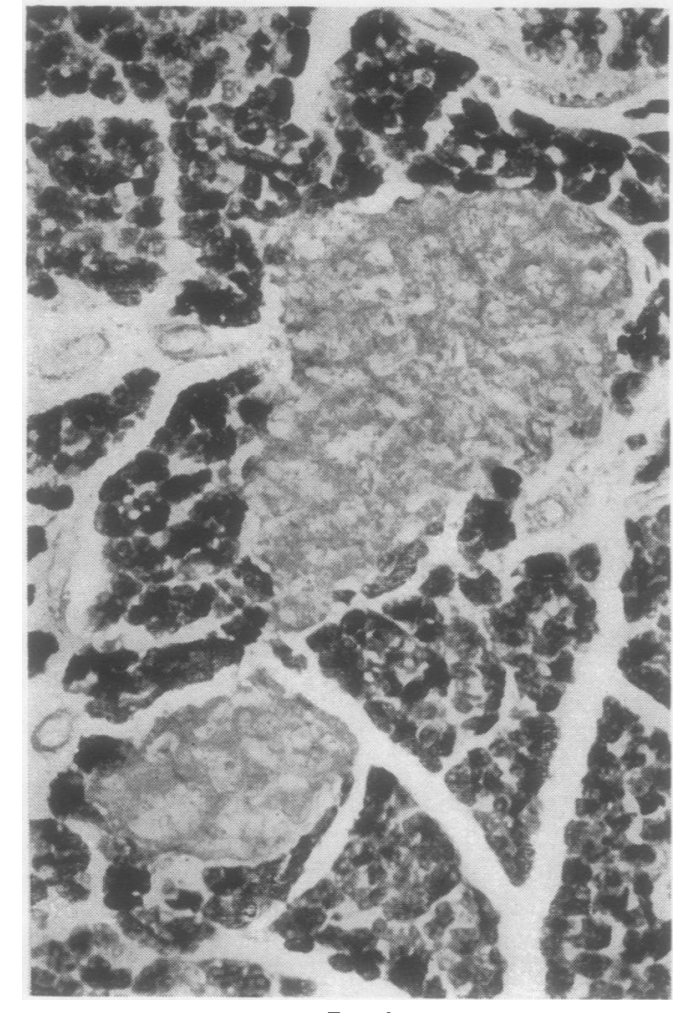

Fig. 2
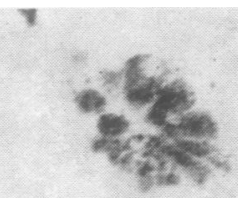

-

Ans

a

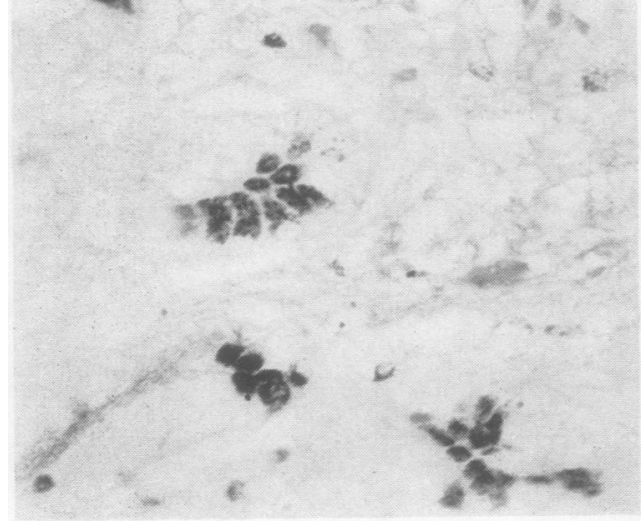

Fio. 3

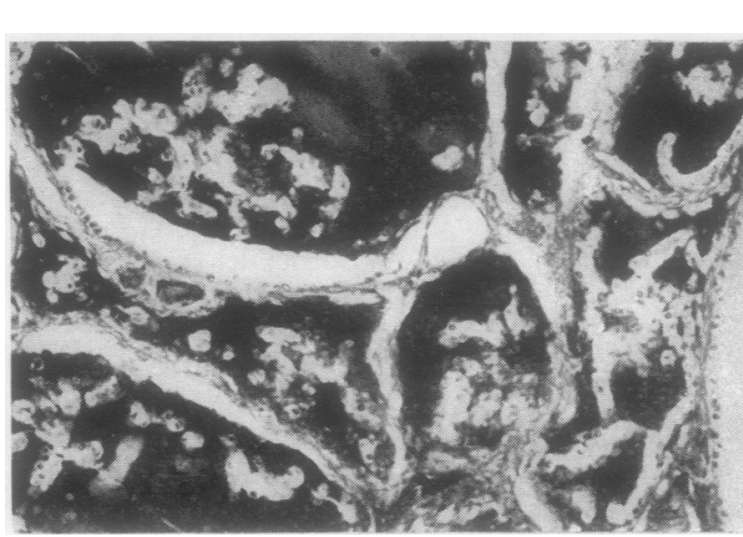

Fig. 4

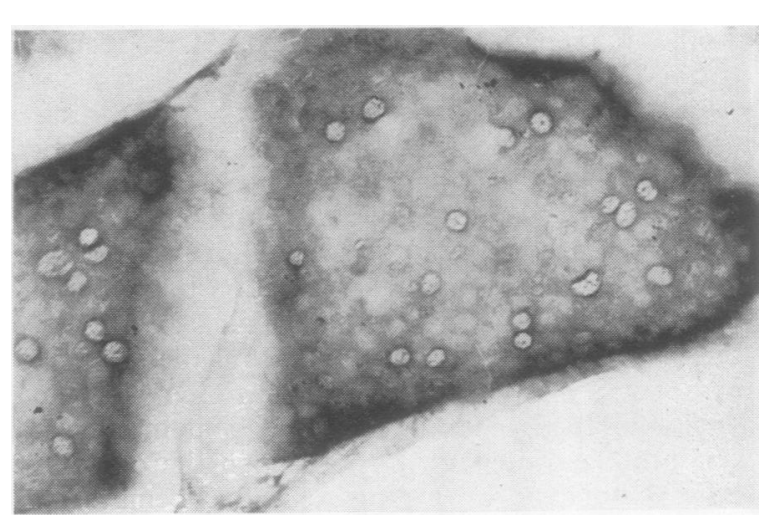

Fig. 5

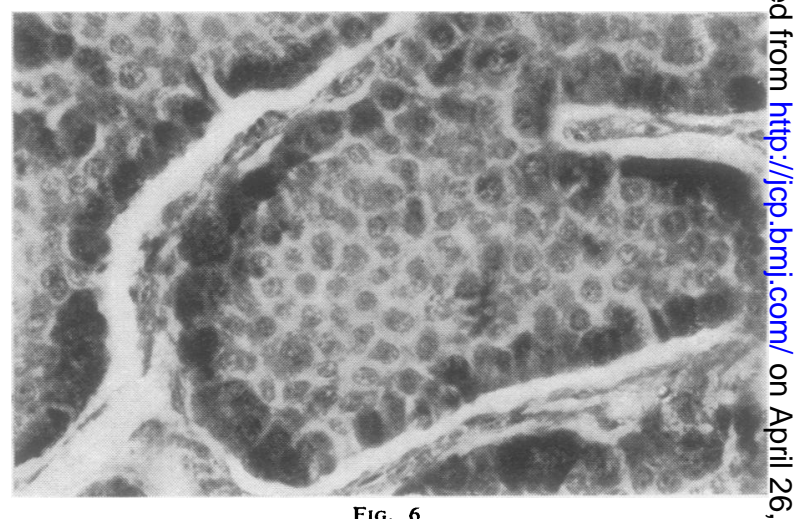

Fig. 6

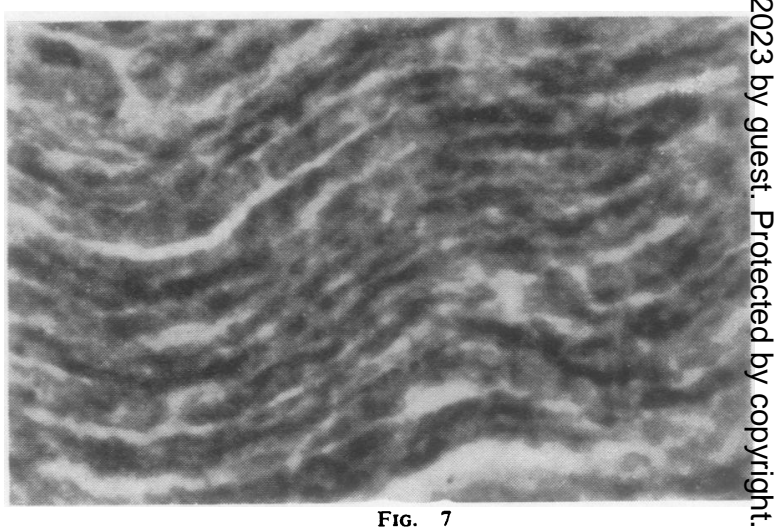


colloid (Fig. 4) is probably related to the relatively high content of tryptophane in all globulins, if not partly to thyroglobulin itself.

In the case of the enterochromaffin system, the argentaffin granules cannot, as reported by Barter and Pearse (1953), be seen in tissues not fixed in formalin. Likewise these argentaffin granules and cells could not be demonstrated with the DABnitrite method after alcoholic fixation. However, in sections of part of a malignant argentaffinoma, fixed in $70 \%$ alcohol, a moderately strong nongranular reaction was obtained with the DABnitrite method in the cytoplasm of cells lining the periphery of most lobules of this tumour (Fig. 5). The distribution of this blue-staining material coincided with that of a strong positive granular reaction seen in formalin-fixed sections of the same carcinoid with the ferric ferricyanide reduction (Fig. 6), direct silver reduction, and alkaline diazonium methods. From this it is inferred that the cytoplasmic material, from which the granules are formed during formaldehyde-fixation, contains a 3-indolyl derivative. This finding is consistent with the histochemical identification of serotonin in the argentaffin granule by Barter and Pearse (1955).

A further interesting finding was the presence of a strong reaction for tryptophane in the "neurokeratin" of the myelin sheaths of peripheral nerves (Fig. 7). "Neurokeratin" is probably formed as an artefact of fixation from the thin unimolecular layers of protein which, as seen

FIG. 2.-Pancreas (rat). Strong reaction in the zymogen granules, but the islet-cells remain unstained. Sulphosalicylic acid fixation. DAB-nitrite, nuclei counterstained with haematoxylin left in its red acid-state, red filter, $\times 135$.

FIG. 3.-Ileum (human). Fairly strong reaction in the Paneth cell granules of the epithelium and also in the cytoplasm of some histiocytes of the lamina propria. Short $(12 \mathrm{hr}$.) formaldehyde fixation. Same technique and filter as in Fig. 2, $\times 360$.

Fig. 4.-Thyroid (rat). Strong reaction in the colloid but not in the follicular cells. Trichloracetic acid-alcohol fixation. Same technique and filter as in Fig. 2, × 135.

FIG. 5.-Malignant argentaffinoma. Moderately strong non-granular reaction in the cytoplasm of cells at the periphery of most lobules. Alcohol fixation. Same technique and filter as in Fig. 2, $\times 330$.

FIG. 6.-Same tumour as in Fig. 5. Strong granular reaction with its maximum intensity, as in Fig. 5 , in cells at the edge of most lobules. Formaldehyde fixation. Ferric ferricyanide reduction reaction, $\times 330$.

FIG. 7.-Sciatic nerve (rat). Strong reaction in the " neurokeratin" of the myelin sheath, but the axis cylinder remains unstained. Trichloracetic acid-alcohol fixation. DAB-nitrite, red filter, $\times 780$. by $x$-ray diffraction and under the electron microscope, alternate with layers of lipid in the cylindroconic units of the myelin sheath of peripheral nerves (Schmitt, 1950 ; Fernández-Morán, 1950).

It is hoped that the DAB-nitrite technique will be of value for the histochemical identification of tryptophane and related compounds. Its value lies in the ease of its performance and its high specificity, since the substance demonstrated, a 3indolyl derivative, forms an essential part of the molecule of the blue pigment formed.

\section{Summary}

A technique for the demonstration of tryptophane and related compounds has been reinvestigated in vitro and applied to histochemical use.

Tryptophane and related 3-indolyl derivatives condense with p-dimethylaminobenzaldehyde and subsequently form a strong blue pigment after oxidation with nitrite.

Alcohol fixatives are recommended; short fixation in formaldehyde may be used ; fixation in chromates should be avoided.

A strong reaction for tryptophane was given by many tissues, in particular, "neurokeratin" of the myelin sheath, the cytoplasm of some neurones, pancreatic zymogen granules, Paneth cell granules, and muscle fibres. A moderate reaction was found in the cytoplasm of some cells of a malignant argentaffinoma.

I am greatly indebted to Professor D. S. Russell for her advice and interest in this work, to Mr. K. Swettenham for technical assistance, and to Mr. A. L. Gallup for the photomicrographs.

\section{Addendum}

Since this paper was submitted for publication a histochemical method for tryptophane has been published by Lillie (1956). This method, which involves exposure for five minutes to $5 \%$ DAB dissolved in a $1: 3$ solution of concentrated hydrochloric and glacial acetic acids, would appear to be equivalent to stage 1 of the reaction described herein, since oxidation by nitrous acid (which greatly increased the sensitivity of our reaction) was not employed by Lillie. This might be tentatively confirmed by the less widespread occurrence of tryptophane in tissues as recorded by this author. 


\section{REFERENCES}

Barter, R., and Pearse, A. G. E. (1953). Nature (Lond.), 172, 810. (1955). J. Path. Bact., 69, 25.

Blanchetière, A., and Romieu, M. (1931). C.R. Soc. Bicl. (Paris), $107,1127$.

Block, R. J., and Bolling, D. (1945). The Amino Acid Composition of Proteins and Foods. Thomas, Springfield, Illinois.

Bourne, G. H. (1951). Cytology and Cell Physinlogy, 2nd ed., p. 351. Clarendon Press, Oxford.

Cuthbertson, W. R., and Phillips, H. (1945). Biochem. J., 39, 7.

Danielli, J. F. (1947). Symp. Soc. exp. Biol. (Cambridge), 1, 101. (1950). Cold Spr. Harb. Symp. quant. Biol., 14, 32.

Fernández-Morán, H. (1950). Exp. Cell Res., 1, 309.

Giral, J., and Laguna, J. (1950). Ciencia (Méx.), 10, 83.

Harvey, D. G., Miller, E. J., and Robson, W. (1941). J. chem. Soc., pt. 1, p. 153.
Kaufmann, B. P., McDonald, M. R., and Gay, H. (1946). Yearb. Carneg. Instn, 45, 158.

Wilson, K., and Wyman, R. (1947). Ibid., 46, 136.

Lillie, R. D. (1956). J. Histochem. Cytochem., 4, 118.

Lison. L. (1936). Histochimie Animale, p. 160, 1st ed. GauthierVillars, Paris.

Pearse, A. G. E. (1953). Histochemistry-Theoretical and Applied, pp. 53, 61, and 421. Churchill, London.

Portner, C., and Hög!, O. (1953). Analyt. Chim. Acta, 8, 29.

Schmitt, F. O. (1950). Biochim. biophys. Acta. 4. $(8$.

Serra, J. A. (1946). Stain Tech., 21, 5.

Spies, J. R., and Chambers, D. C. (1948). Analyt. Chem., 20, 30.

Toennies, G., and Homiller, R. P. (1942). J. Amer. chem. Soc., 64, 3054 . 